\title{
INFORMATIONAL ENTROPY OF FRACTAL RIVER NETWORKS
}

Pierluigi Claps, Mauro Fiorentino and Giuseppe Oliveto

\author{
Dipartimento di Ingegneria e Fisica dell'Ambiente \\ Università della Basilicata \\ Via della Tecnica 3 \\ 85100, Potenza (Italy)
}




\begin{abstract}
Informational entropy of river networks, as defined by Fiorentino and Claps (1992a), proved to be a useful tool in the interpretation of several properties exhibited by natural networks. In this paper, self-similar properties of river networks are taken as the starting point for investigating analogies and differences between natural networks and geometric fractal trees, comparing their variability entropy with parameters of both classes of networks. Attention is directed particularly to relations between entropy and Horton order and entropy and topological diameter of subnetworks. Comparisons of these relations for fractals and natural networks suggest that network entropy can contribute to clarify important points concerning self-similar properties of river networks. Moreover, the estimation of the fractal dimension of branching for natural networks can be considerably improved using the relation between entropy and Horton order throughout the network.
\end{abstract}

\title{
1. INTRODUCTION: INFORMATIONAL ENTROPY OF RIVER NETWORKS
}

The informational entropy of river networks was defined by Fiorentino and Claps $(1992 a)$ as:

$$
\mathrm{S}=-\sum_{\delta=1}^{\Delta} \mathrm{p}_{\delta} \ln \mathrm{p}_{\delta}
$$

consistently with the definition given by Shannon (1948) in information theory, where $\mathrm{p}_{\delta}^{\prime} \mathrm{s}$ the relative probabilities related to a given "state" $\delta$. It can be shown (e.g. Wannier, 1966) that the informational definition of entropy is equivalent to its definition given in statistical- 
mechanics, in which entropy is proportional to the relative probability of a state of a thermodynamical system.

In the definition of informational entropy of a river network, the network is considered as a system in which stream segments (links) are the elements whose placement characterize the system configuration. A network link is the path connecting two junctions and the topological distance from the outlet, i.e. the number of consecutive links forming the shortest path from its upstream node to the outlet, corresponds to the state $\delta$ in which the link is placed (see figure 1). The selection of $\delta$ as the state variable in the relation (1) was further substantiated by Fiorentino et al. (1993). The total number of states is the network topological diameter $\Delta$, corresponding to the maximum topological distance from the outlet. If one disregards the length of links, as is assumed in this paper, the network configuration is completely determined by the topological width function which is the diagram of the relative frequency $\mathrm{p}_{\delta}$ of the links as a function of the topological distance $\delta$. The maximum entropy for a given topological diameter is attained with uniform width function (i.e. same number of links at each topological distance) and is expressed as $\mathrm{S}_{\max }=\ln \Delta$.

Under the hypothesis of uniform energy expenditure, Fiorentino et al. (1993) obtained analytical expressions of channel profiles by maximizing entropy subject to the knowledge of the average elevation of the river network. Also some important scaling properties of channels, such as slope-area and stream power-area relationships, were derived, with scaling factors depending on the fractal dimension $\mathrm{D}$ of the branching process of the channel network.

Moreover, the informational entropy of river network was empirically found (Fiorentino et al., 1993) to vary with marked regularity with network characteristics, particularly Horton order, magnitude and topological diameter. In this paper, a specific investigation of the above regularities is presented, looking at properties of both fractal and natural networks with regard to entropy.

To take advantage of the fractal nature of river networks, first hypothesized by Mandelbrot (1983) and later confirmed by several authors with different approaches, synthetic 
fractal networks are introduced here and their properties are exploited to achieve analytical expressions of the entropy as a function of parameters of their configuration. Entropy properties exhibited by natural river networks are then compared with the properties of the entropy of some geometric fractal trees, resulting in interesting interpretations of empirical findings.

\section{DEFINITION OF FRACTAL PLANE TREES AND THEIR INFORMATIONAL ENTROPY}

\subsection{A model of fractal plane trees}

Fractal plane trees are fractal (self-similar) objects, that can be defined as "having a shape made of parts similar to the whole in some way" (Mandelbrot, 1986). Fractal objects result from repeated generations, starting from an initiator, which is a set of segments, and using a generator, which is a different set of segments. In the construction of the fractal set first the initiator is substituted with the generator and then each segment of the generator becomes an initiator and is substituted again, in a recursive way (e.g. Feder, 1988, p. 16). If only selected segments of the generator become initiators, the resulting tree is a non-uniform fractal. However, our approach is restricted to uniform fractals.

Fractal plane trees can be obtained with an initiator made of a unit-length segment and a tree-like generator, made of equal shorter segments whose length is $\eta$ (Mandelbrot, 1983, pp.72-73).

After a finite number $m$ of generations the resulting tree, which we will call $m$-structure, has finite Euclidean length and is indeed a prefractal, since fractal object have infinite length. The number of segments of an $m$-structure is $\mathrm{M}_{m}=\mathrm{M}_{1}{ }^{m}$, with $\mathrm{M}_{1}$ as the number of segments of the generator, and its segment length is $\zeta_{m}=\eta^{m}$ (see figure 2). In this paper, generators are 
taken such that the longest path is straight and segments form only right angles. This does not affect generality and allows us to obtain that the number of partitions $1 / \eta$ of the initiator equals $\Delta_{1}$, which is the topological diameter of the generator tree. After $m$ generations, the topological diameter is:

$$
\Delta_{m}=\Delta_{1}^{m}
$$

The Euclidean length $\mathrm{L}(\zeta)=\mathrm{M}(\zeta) \zeta$ of the self-similar geometric set depends on the unit of measure $\zeta$ and of the number $\mathrm{M}(\zeta)$ of segments necessary to cover the set, both changing with each generation. The fractal measure (i.e. invariant with respect to the index of generation) $\mathrm{L}=\mathrm{M}(\zeta) \zeta^{\mathrm{D}}$ does not change with generations because of the way the fractal dimension $\mathrm{D}$ is defined (e.g. Feder, 1988, p.19):

$$
\mathrm{D}=-\frac{\ln \mathrm{M}_{1}}{\ln \eta}
$$

Considering that various fractal dimensions have been introduced for river networks (e.g. Liu, 1992; Beer and Borgas, 1993), this fractal dimension is to be considered as only due to the branching process and is better specified as the network similarity dimension.

\subsection{Informational entropy of fractal trees}

The topological width function of fractal trees can be readily obtained recursively as a function of $m$ by first determining the number $\mathrm{W}_{m}(\mathrm{j})\left(\mathrm{j}=1, . ., \Delta_{m}\right)$ of links at the topological distance $j$ from the outlet. To this end, it is convenient to separate $\mathrm{W}_{m}(\mathrm{j})$ in $\Delta_{1}$ classes, each of length $\Delta_{m-1}$. The reason for this is that, after the generation transforming an $(m-1)$-structure into a $m$-structure, this latter contains as many $(m-1)$-structures as the number of segments of the generator. The network diameter will then become $\Delta_{m}=\Delta_{1}{ }^{m}=\Delta_{1} \Delta_{m-1}$, leading to $\Delta_{1}$ classes. In the generic $\Delta_{\mathrm{i}}$ class, $\mathrm{W}_{m}(\mathrm{j})$ is made up of as many $(m-1)$-structures as the number of segments of the generator at the level $i$, leading to: 


$$
\begin{array}{ll}
\mathrm{W}_{m}\left(1, \ldots, \Delta_{m-1}\right)=\mathrm{W}_{1}(1) \cdot \mathrm{W}_{m-1}\left(1, \ldots \Delta_{m-1}\right)=1 \cdot \mathrm{W}_{m-1}\left(1, \ldots \Delta_{m-1}\right) & \text { class } 1 \\
\mathrm{~W}_{m}\left(1 \cdot \Delta_{m-1}+1, \ldots, 2 \cdot \Delta_{m-1}\right)=\mathrm{W}_{1}(2) \cdot \mathrm{W}_{m-1}\left(1, \ldots \Delta_{m-1}\right) & \text { class } 2 \\
\mathrm{~W}_{m}\left(\left(\Delta_{1}-1\right) \cdot \Delta_{m-1}+1, \ldots, \Delta_{1} \cdot \Delta_{m-1}\right)=\mathrm{W}_{1}\left(\Delta_{1}\right) \cdot \mathrm{W}_{m-1}\left(1, \ldots \Delta_{m-1}\right) & \text { class } \Delta_{1}
\end{array}
$$

Since the generic class $r$ of the function $\mathrm{W}_{(m)}(\mathrm{j})$ corresponding to the $m$-th multiplication has $\Delta_{m-1}$ topological levels:

$$
\mathrm{W}_{(m)}(\mathrm{j}), \mathrm{j}=(\mathrm{r}-1) \cdot \Delta_{m-1}+1, \ldots, \mathrm{r} \cdot \Delta_{m-1}
$$

it is convenient to introduce the following notation:

$$
\mathrm{rW}_{(m)}(\mathrm{i}), \quad \mathrm{i}=1 \ldots \Delta_{m-1}
$$

where $i$ is the relative topological level. Hence, probabilities associated to the topological distances in the generic class of the width function are:

$$
r \mathrm{P}_{m}(\mathrm{i})=\frac{r \mathrm{~W}_{m}(\mathrm{i})}{\mathrm{M}_{\mathrm{m}}}, \quad \mathrm{i}=1 \ldots \Delta_{m-1} .
$$

Considering that

$$
r \mathrm{~W}_{m}(\mathrm{i})=\mathrm{W}_{1}(r) \cdot \mathrm{W}_{m-1}(\mathrm{i}), \quad \mathrm{i}=1 \ldots \Delta_{m-1}
$$

and that after $m$ generations the total number of segments is $\mathrm{M}_{m}=\mathrm{M}_{1}{ }^{m}$, the probability is:

$$
r \mathrm{P}_{m}(\mathrm{i})=\frac{\mathrm{W}_{1}(\mathrm{r})}{\mathrm{M}_{1}} \frac{\mathrm{W}_{\mathrm{m}-1}(\mathrm{i})}{\mathrm{M}_{\mathrm{m}-1}}, \quad \mathrm{i}=1 \ldots \Delta_{m-1}
$$

and can be expressed as:

$$
{ }^{\mathrm{r}} \mathrm{P}_{m}(\mathrm{i})=\frac{\mathrm{W}_{1}(\mathrm{r})}{\mathrm{M}_{1}} \mathrm{P}_{\mathrm{m}-1}(\mathrm{i}), \quad \mathrm{i}=1 \ldots \Delta_{m-1}
$$

With reference to the $m$-structure, the expression (1) defining the informational entropy can be expanded in order to show all components of the summation:

$$
\mathrm{S}_{m}=\sum_{\mathrm{j}=1}^{\Delta_{m-1}} \mathrm{P}_{m}(\mathrm{j}) \ln \mathrm{P}_{m}(\mathrm{j})+\sum_{\mathrm{j}=\Delta_{m-1}+1}^{2 \cdot \Delta_{m-1}} \mathrm{P}_{m}(\mathrm{j}) \ln \mathrm{P}_{m}(\mathrm{j})+. .+\sum_{\mathrm{j}=\left(\Delta_{1}-1\right) \cdot \Delta_{m-1}+1}^{\Delta_{1} \cdot \Delta_{m-1}} \mathrm{P}_{m}(\mathrm{j}) \ln \mathrm{P}_{m}(\mathrm{j})
$$


The summation term for the generic class $r$ is:

$$
\sum_{\mathrm{i}=1}^{\Delta_{\mathrm{m}-1} r} \mathrm{P}_{m}(\mathrm{i}) \ln { }^{r} \mathrm{P}_{m}(\mathrm{i})
$$

and considering that equation (4) gives

$$
\ln \mathrm{r}_{m}(\mathrm{i})=\ln \mathrm{P}_{m-1}(\mathrm{i})+\ln \frac{\mathrm{W}_{1}(\mathrm{r})}{\mathrm{M}_{1}}
$$

one can rewrite (6) as:

$$
\sum_{\mathrm{i}=1}^{\Delta_{\mathrm{m}-1}} \frac{\mathrm{W}_{1}(\mathrm{r})}{\mathrm{M}_{1}}\left[\mathrm{P}_{\mathrm{m}-1}(\mathrm{i}) \ln \mathrm{P}_{\mathrm{m}-1}(\mathrm{i})+\mathrm{P}_{\mathrm{m}-1}(\mathrm{i}) \ln \frac{\mathrm{W}_{1}(\mathrm{r})}{\mathrm{M}_{1}}\right]
$$

Expanding all terms in parenthesis we get:

$$
\frac{\mathrm{W}_{1}(\mathrm{r})}{\mathrm{M}_{1}} \sum_{\mathrm{i}=1}^{\Delta_{\mathrm{m}-1}} \mathrm{P}_{\mathrm{m}-1}(\mathrm{i}) \ln \mathrm{P}_{\mathrm{m}-1}(\mathrm{i})+\frac{\mathrm{W}_{1}(\mathrm{r})}{\mathrm{M}_{1}} \ln \frac{\mathrm{W}_{1}(\mathrm{r})}{\mathrm{M}_{1}} \sum_{\mathrm{i}=1}^{\Delta_{\mathrm{m}-1}} \mathrm{P}_{\mathrm{m}-1}(\mathrm{i})
$$

where the first summation represents the entropy $\mathrm{S}_{m-1}$ of the $(m-1)$-structure and the second summation equals 1. Substituting the generic term (7) into expression (5) the resulting equation is

$$
\mathrm{S}_{\mathrm{m}}=\mathrm{S}_{\mathrm{m}-1} \sum_{\mathrm{r}=1}^{\Delta_{1}} \frac{\mathrm{W}_{1}(\mathrm{r})}{\mathrm{M}_{1}}+1 \cdot \sum_{\mathrm{r}=1}^{\Delta_{1}} \frac{\mathrm{W}_{1}(\mathrm{r})}{\mathrm{M}_{1}} \ln \frac{\mathrm{W}_{1}(\mathrm{r})}{\mathrm{M}_{1}}
$$

from which, considering that $\sum \mathrm{W}_{1}=\mathrm{M}_{1}$, finally results:

$$
\mathrm{S}_{\mathrm{m}}=\mathrm{S}_{\mathrm{m}-1}+\mathrm{S}_{1}
$$

that by recursion leads to the straightforward equation:

$$
\mathrm{S}_{m}=m \mathrm{~S}_{1}
$$

It is interesting to compare this result to the variation of entropy with growth of maximum-entropy and minimum-entropy-production tree structures. Fiorentino and Claps (1992b) introduced these tree structures, which present opposite characteristics. A maximum-entropy tree (figure 3.a) grows in such a way to maintain uniformity in the width function (which is indeed asymptotical). In this growth, Horton order does not increase while entropy does. A minimum entropy-production tree (figure 3.b) is built in order to minimize 
the increase of entropy with its growth. This is obtained maximizing the increase in the number of links for increasing topological diameter (at each growth stage every source bifurcates producing also an increase in the network Horton order); for such a structure, entropy tends asymptotically to a constant. Both tree structures are non-uniform fractals.

In the next section, the patterns shown by these two structures are compared to the variation of entropy with respect to some parameters of natural and fractal networks.

\section{PROPERTIES OF FRACTAL AND NATURAL NETWORKS WITH REGARD TO ENTROPY}

\subsection{Informational entropy and Horton order of subnetworks}

In natural networks the average entropy of subnetworks of the same Horton order was found (Fiorentino et al., 1993) to vary linearly with the order. In particular, the equation

$$
\mathrm{S}_{\Omega}=c(\Omega-1)
$$

was found, with very high correlation coefficient $(>0.99)$ and with $c$ very close to 1. Coefficient $c$ is the rate of increase of entropy with the order, that corresponds to a rate of entropy production. Also $c$ is an estimate of the average entropy of second-order subnetworks.

Analyzing synthetic fractal trees in a hortonian framework it is easy to determine the order of subnetworks, since each generation transforms first-order links into trees equal to the generator increasing the network order of an amount equal to $\left(\Omega_{1}-1\right)$. After $m$ generations the tree is an $m$-structure with order:

$$
\Omega_{m}=1+m\left(\Omega_{1}-1\right)
$$

Hortonian subnetworks of an $m$-structure can be or can be not $j$-structure themselves, depending on the order $\Omega_{1}$ of the generator. 
By substituting in (10) $m$ as taken from (8), entropy of $m$-structure subnetworks of order $\Omega_{m}$ is obtained as:

$$
\mathrm{S}_{\Omega_{m}}=\frac{\Omega_{m}-1}{\Omega_{1}-1} \cdot \mathrm{S}_{1}
$$

For generators of order 2, all Hortonian subnetworks are $j$-structures, so (11) can be written as:

$$
\mathrm{S}_{\Omega}=(\Omega-1) \mathrm{S}_{1}
$$

which is equal to relation (9) found for natural networks. Therefore it becomes apparent the meaning of the coefficient $c$ in (9), as the entropy of an equivalent generator producing the fractal structure of branching in the river network.

In this paper, fractal networks are built using four kinds of trivalent (i.e. three segments joining into each node) generators (figure 4) and data from eight natural drainage networks of southern Italy basins were used, with characteristics reported in table 1.

Figure 5 shows the relative position of the straight lines corresponding to $(\Omega, S)$ relations for natural and fractal networks. Lines related to natural networks were obtained (Fiorentino et al., 1993) by linear regression between Horton order and average entropy of subnetworks of equal order. All subnetworks are considered at fixed resolution.

It can be noted that natural basins behaves as fractal structures mostly intermediate between these obtained from generators 4.a and 4.b. Even though the use of generators of order $\Omega_{1}$ greater than 2 is awkward, because there is no explicit expressions for the entropy of subnetworks which are not $j$-structures, a third order generator (figure 4.d) was considered. It can be regarded as a structure intermediate between the 4.a and 4.b, as confirmed by the position of its $(\Omega, S)$ curve. In this case, equation (11) can be applied only for Hortonian subnetworks of odd order, which are all j-structures. Subnetworks of even order are still equal throughout the network but their entropy can only be computed through the definition (1). It is also interesting to comment the position of curves relative to maximum-entropy and 
minimum-entropy-production networks: both curves constitutes bounds of the region of existence of real-world curves and show the two related networks to have characteristics very different, in a Hortonian viewpoint, from those of natural and fractal networks.

\subsection{Informational entropy and topological diameter of subnetworks}

From what was shown previously, the topological diameter $\Delta$ of a subnetwork seems to be a scale factor of the object by an entropic viewpoint, first of all because $\ln \Delta$ is the entropy upper bound. Yet, it is less apparent if some regularity exists in the $(\mathrm{S}, \Delta)$ relation for subsets of a network, either natural or fractal. To investigate this, Claps and Oliveto (1993) examined the relation existing between the two variables for all subnetworks of the eight basins of table 1. Linear relations with very high correlation coefficients $(>0.99)$ were found between $\mathrm{S}$ and $\ln \Delta$.

Moving to synthetic fractal networks, equation (2) provides the topological diameter of all $m$-structures, so that eliminating $m$ between (2) and (8) one obtains:

$$
\mathrm{S}_{\Delta_{\mathrm{m}}}=\frac{\ln \Delta_{m}}{\ln \Delta_{1}} \cdot \mathrm{S}_{1}
$$

which is the same kind of equation found for natural basins.

It is interesting to comment how close natural basins and fractal networks curves are to the case of maximum-entropy structures, for which $\mathrm{S}_{\Delta}=\ln \Delta$. Regarding this, observation of figure 6 reveals that the lines relative to both fractal and natural networks deviate only slightly from the diagonal representing the case of maximum-entropy trees. This appears in contrast with the patterns observed in fig.5. However, it is to be considered that Horton order doesn't give any information on maximum-entropy trees, since the order $\Omega$ is 2 independently of the tree configuration. 
The dotted lines in fig.6, representing the regression lines found for natural basins, are again comprised between the lines of the two fractal trees obtained from generators 4.a and 4.b.

Figure 6 also shows the curve relative to the Peano fractal network (e.g. Marani et al., 1991) which is a tetravalent structure (four links joining into each node) with fractal dimension 2 (is a plane-filling curve). This fractal tree occupies a completely different region of the $(\mathrm{S}, \Delta)$ plane with respect to the other trivalent objects. This constitutes an additional confirmation of the doubts expressed by Rodriguez-Iturbe et al. (1992), Fiorentino and Claps (1992b) and Rinaldo et al. (1992), on the use of this fractal tree as a surrogate of natural networks, for which it was proposed mainly because of its space-filling nature.

\section{ESTIMATION OF RIVER NETWORK FRACTAL DIMENSION USING ENTROPY}

Synthetic fractal trees are completely defined by their fractal dimension (3) and, given the results of the previous sections, by the informational entropy of their generator. A comparison of the definition (3) with the equation

$$
\mathrm{D}=\frac{\ln \mathrm{R}_{\mathrm{B}}}{\ln \mathrm{R}_{\mathrm{L}}}
$$

introduced by La Barbera and Rosso (1989) for the river network fractal dimension, suggests an analogy between $\mathrm{M}_{1}$ and the Horton bifurcation ratio $\mathrm{R}_{\mathrm{B}}$ and between $\Delta_{1}$ and the Horton length ratio $R_{L}$.

Claps and Oliveto (1993) have shown that this analogy is asymptotically an equality for fractal networks with $\Omega_{1}=2$ and that estimation of the similarity dimension $\mathrm{D}$ of fractal networks by (14) is correct only for trees with an adequately high generation index $\mathrm{m}$. The latter result is valid for whatever $\Omega_{1}$. The sensitivity of the equation (14), as a function of the 
network Horton order, for the estimate of the fractal dimension of branching was also shown by Beer and Borgas (1993) with reference to a natural network.

The question arise whether the similar patterns exhibited by fractal and natural networks with reference to their informational entropy can be useful in reconsidering the estimation of the network similarity dimension. As a matter of fact, both $\mathrm{D}$ and $\mathrm{S}_{1}$ are unique for each specific fractal tree, particularly if simple generators are considered. So, a simple answer to the above question can be given by the construction of a regression link between the two variables.

Using only fractal structures with $\Omega_{1}=2$, including those of figure 2 and trees with increasing topological diameter, the equation

$$
\mathrm{D}=1.476-0.240 \ln \mathrm{S}_{1}
$$

was obtained by regression, with correlation coefficient $\mathrm{R}=0.999$. This equation can be used to estimate $\mathrm{D}$ once $\mathrm{S}_{1}$ is known.

Equation (15) was applied to the eight natural basins considered, with $\mathrm{S}_{1}$ evaluated by the regression between $\mathrm{S}_{\Omega}$ and $\Omega$ (equation (12)). Resulting estimates of $\mathrm{D}$, reported in table 2 , are much more stable around their mean in comparison to the estimates obtained through (14); this could be interpreted as an index of robustness of the estimation method.

\section{FINAL REMARKS}

Some relations between the informational entropy of the network and parameters characterizing the network structure have been investigated in this paper with regard to synthetic fractal trees. These relations, established in analytical form, were found perfectly matching empirical relations obtained in previous papers on natural networks. Therefore, the fractal nature of river networks is substantiated by these analogies and used to better 
understand patterns of variability of some parameters as functions of the informational entropy.

Some particular findings are worth emphasizing:

1) the entropy of fractal and natural networks is linearly related, in average, to the Horton order. The slope $S_{1}$ of the straight line represents the entropy of the elementary structure from which the self-similar network can be considered to be generated.

2) the logarithm of the topological diameter is linearly related to entropy, with slope close to 1. So, natural networks can be considered as quasi maximum-entropy structures;

3) both $S_{1}$ and the fractal dimension $D$ are unique features of the self-similar networks. Hence, the relation linking these two variables permits an interesting new method for estimating the fractal dimension of the branching for natural networks.

\section{Acknowledgments}

This work was supported by funds granted by the Italian Ministero della Ricerca Scientifica e Tecnologica, Progetto 40\% "Processi Idrologici Fondamentali". Comments and suggestions by an anonymous reviewer are acknowledged and appreciated.

\section{REFERENCES}

Beer, T. and Borgas, M., 1993. Horton's laws and the fractal nature of streams. Water Resources Research, 29 (5): 1475-1487.

Claps,P. and Oliveto, G., 1993. Entropia informativa delle reti frattali e proprietà di autosomiglianza dei reticoli di drenaggio naturali, (in italian). Dipartimento di Ingegneria e Fisica dell'Ambiente, Università della Basilicata, Potenza, Italy.

Feder, J., 1988. Fractals. Plenum Press, New York, 283 pp. 
Fiorentino, M. and Claps, P., 1992a. On what can be explained by the entropy of a channel network. In: V.P. Singh and M. Fiorentino (Editors). Entropy and Energy Dissipation in Water Resources. Kluwer, Dordrecht, The Netherlands, pp. 139-154.

Fiorentino, M. and Claps P., 1992b. Un'analisi di alcuni fenomeni idrologici a scala di bacino mediante l'uso del concetto di entropia, (in italian). Proc. XXIII Convegno di Idraulica e Costruzioni Idrauliche. Dept. of Civil Eng., Univ. of Florence, Florence, D.193-D.207.

Fiorentino, M., Claps, P. and Singh, V.P., 1993. An entropy-based morphological analysis of river-basin networks. Water Resources Research, 29(4): 1215-1224.

La Barbera, P. and Rosso, R., 1989. On the fractal dimension of stream networks. Water Resources Research, 25(4): 735-741.

Liu, T., 1993. Fractal structure and properties of stream networks. Water Resources Research, 28 (11): 2981-2988.

Mandelbrot, B.B., 1983. The Fractal Geometry of Nature, W.H. Freeman, New York, 468 pp.

Mandelbrot, B.B., 1986. Self-affine fractal sets. in: L. Pietronero and E. Tosatti (Editors). Fractals in Physics. North-Holland, Amsterdam, pp. 3-28.

Marani, A., Rigon, R. and Rinaldo, A., 1991. A Note on Fractal Channel Networks. Water Resources Research, 27(12): 3041-3049.

Rinaldo, A., Rodriguez-Iturbe, I., Rigon, R., Bras, R.L., Ijjasz-Vasquez, E. and Marani, A., 1992. Minimum Energy And Fractal Structures Of Drainage Networks. Water Resources Research, 28 (9): 2183-2195.

Rodriguez-Iturbe, I., Rinaldo, A., Rigon, R., Bras, R.L., Ijjasz-Vasquez E. and Marani, A., 1992. Fractal structures as least energy patterns: the case of river networks. Geophysical Research Letters, 19 (9): 889-892.

Shannon, C. E., 1948. The mathematical theory of communications, I and II. Bell System Technical Journal, 27: 379-423.

Wannier, G.H., 1966. Statistical Physics. J. Wiley, New York, 532 pp. 


\begin{tabular}{lcccccccccc}
\hline Basin & $A$ & Dr. Dns. & M. L. & $n$ & $H$ & $E$ & $\Delta$ & $\Omega$ & $R_{B}$ & $R_{L}$ \\
& $\left(K^{2}\right)$ & $\left(k^{-1}\right)$ & $(K m)$ & & $(m)$ & $(m)$ & & & & \\
\hline Arcidiaconata & 123.9 & 2.24 & 23.04 & 254 & 657 & 301 & 50 & 5 & 4.12 & 2.39 \\
Lapilloso & 28.5 & 2.34 & 11.56 & 72 & 394 & 229 & 36 & 4 & 4.34 & 2.28 \\
Vulgano & 94.1 & 2.08 & 22.08 & 193 & 663 & 370 & 37 & 5 & 3.79 & 2.26 \\
S.Maria & 58.1 & 2.26 & 15.53 & 159 & 226 & 144 & 48 & 5 & 3.72 & 2.57 \\
Salsola & 44.1 & 2.24 & 14.21 & 100 & 513 & 270 & 31 & 5 & 3.28 & 2.29 \\
Casanova & 57.3 & 2.20 & 15.79 & 123 & 524 & 290 & 26 & 5 & 3.44 & 2.55 \\
Celone S.V. & 92.5 & 2.07 & 27.59 & 181 & 715 & 362 & 42 & 5 & 3.83 & 2.73 \\
Celone a P.F. & 233.5 & 1.55 & 48.61 & 292 & 861 & 405 & 53 & 5 & 4.1 & 2.74 \\
\hline
\end{tabular}

Tab. 1. Some characteristics of the Southern Italy basins considered. A (drainage area); Dr.Dns. (drainage density); M. L. (mainstream length); $n$ (magnitude); $H$ (total elevation drop of the main stream); $E$ (average basin elevation relative to the outlet); $\Delta$ (topological diameter); $\Omega$ (Horton order); $R_{B}$ (Horton bifurcation ratio); $R_{L}$ (Horton length ratio). 


\begin{tabular}{lccc}
\hline Basin & $S_{1}$ & $\ln \mathrm{R}_{\mathrm{B}} / \ln \mathrm{R}_{\mathrm{L}}$ & $\mathrm{D}$ \\
\hline Lapilloso & 1.081 & 1.78 & 1.43 \\
Celone F.S. & 0.981 & 1.40 & 1.47 \\
Arcidiaconata & 0.952 & 1.63 & 1.48 \\
Celone S.V. & 0.953 & 1.34 & 1.49 \\
Vulgano & 0.880 & 1.64 & 1.49 \\
Salsola & 0.837 & 1.43 & 1.51 \\
Casanova & 0.859 & 1.32 & 1.53 \\
S.Maria & 1.018 & 1.39 & 1.49 \\
\hline \multirow{4}{*}{} & & & \\
& Average & 1.49 & 1.49 \\
& Std. Dev. & 0.169 & 0.029 \\
\hline
\end{tabular}

Tab. 2. Ln $R_{B} / \ln R_{L}$ and $D$ estimated by equation (15) for the natural networks in Table 1. Values of $S_{1}$ needed in equation (15) are estimated by regression with the order (equation (12)). 


\section{FIGURE CAPTIONS}

Fig. 1. Definition sketch of a simplified drainage network formed by links, external nodes (sources) and internal nodes. The Horton order $\omega$ of each link is reported in parenthesis. In this network the magnitude $\mathrm{n}$ (number of sources) is 6 , the topological diameter $\Delta$ is 5 , the Horton order $\Omega$ is 3 .

Fig. 2. Generation of a fractal tree. Parameter $m$ is the generation index. The structure with $m=1$ is the generator. The initiator is a unit-length segment.

Fig. 3. Representation of non-uniform fractal networks. (a) maximum-entropy network $\left(\mathrm{R}_{\mathrm{b}}=\Delta, \Omega=\right.$ constant $\left.=2\right) ;(\mathrm{b})$ minimum-entropy production network $\left(\mathrm{R}_{\mathrm{b}}=2, \Omega=\Delta\right)$.

Fig. 4. Generators of the fractal networks considered: (a) $\mathrm{M}_{1}=3, \eta=1 / 2, D=1.585, \mathrm{~S}_{1}=0.637$, $\Omega_{1}=2 ;$ (b) $\mathrm{M}_{1}=5, \eta=1 / 3, \mathrm{D}=1.465, \mathrm{~S}_{1}=1.055, \Omega_{1}=2 ;$ (c) $\mathrm{M}_{1}=7, \eta=1 / 4, \mathrm{D}=1.404, \mathrm{~S}_{1}=1.352, \Omega_{1}=2$; (d) $\mathrm{M}_{1}=7, \eta=1 / 5, \mathrm{D}=1.490, \mathrm{~S}_{1}=1.516, \Omega_{1}=3$.

Fig. 5. Empirical and theoretical linear relations between Horton orders and average entropy of subnetworks. Networks are identified as follows: maximum-entropy network $(\cdots 0 \cdots)$; minimum-entropy production network (-•-•-• ); fractal network 4.a (-o- $)$; fractal network 4.b ( $-\mathrm{x}-)$; fractal network 4.c ( -+-$)$; fractal network 4.d (-*- ); natural networks $(\cdots \cdots \cdots)$. .

Fig. 6. Empirical and theoretical linear relations between average entropy of subnetworks with diameter $\Delta$ and $\ln \Delta$. Networks are identified as follows: fractal networks 4.a, 4.b, 4.c' (— (-•-•-- ); minimum-entropy-production network ( $-\mathrm{O}-)$; Peano basin $\left(-+-{ }_{-}\right)$. 


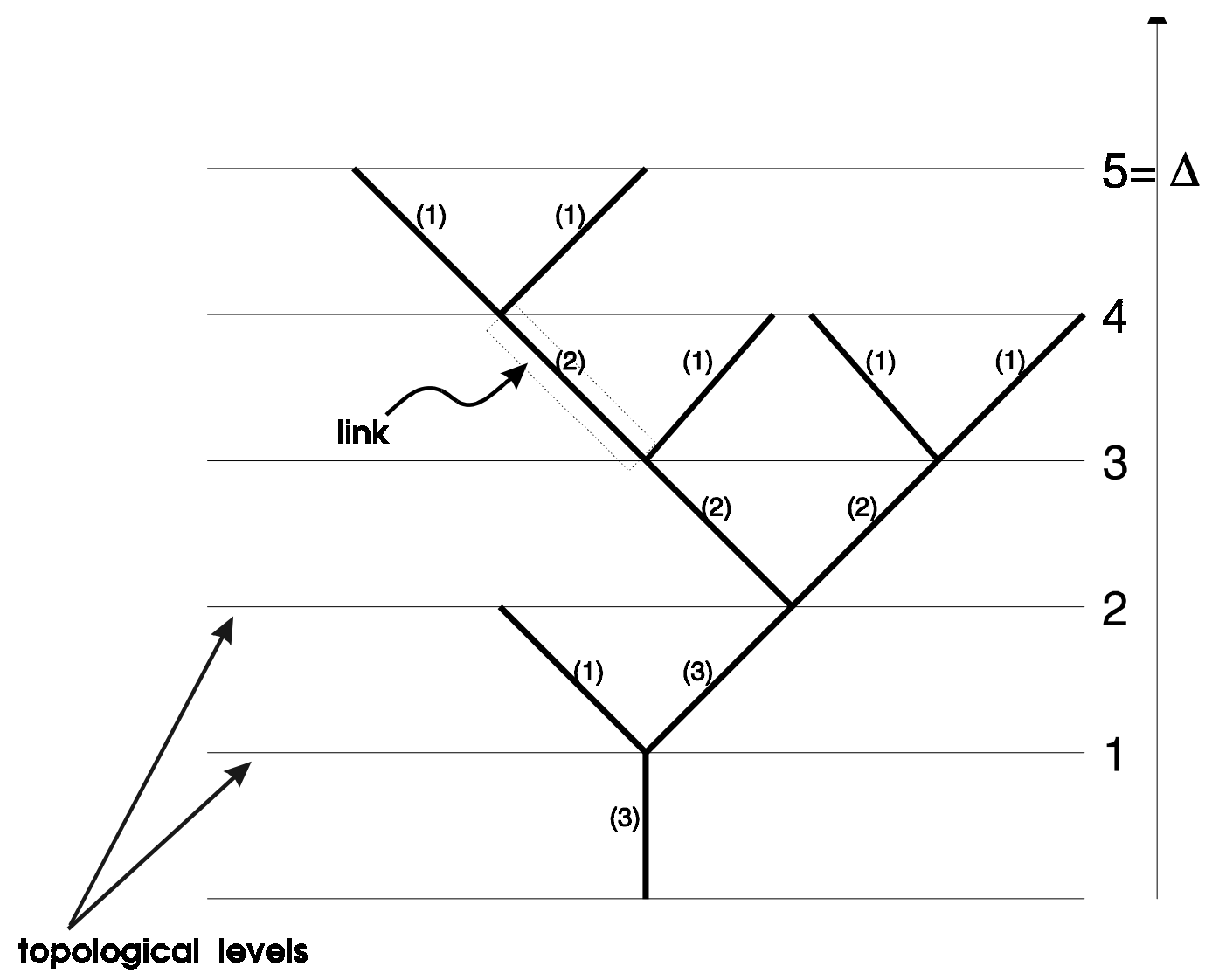

Figure 1 


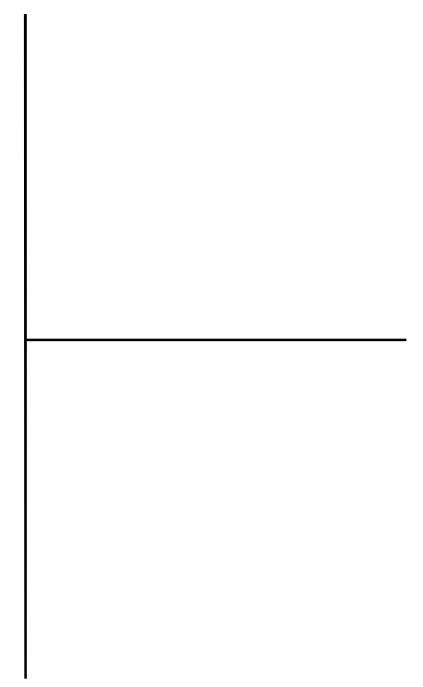

$m=1$

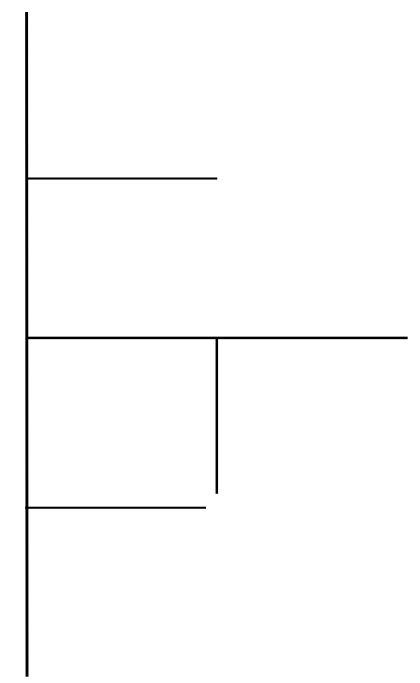

$m=2$

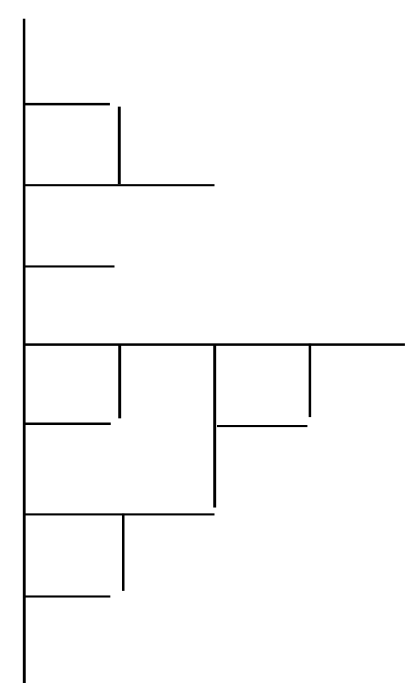

$m=3$

Figure 2 


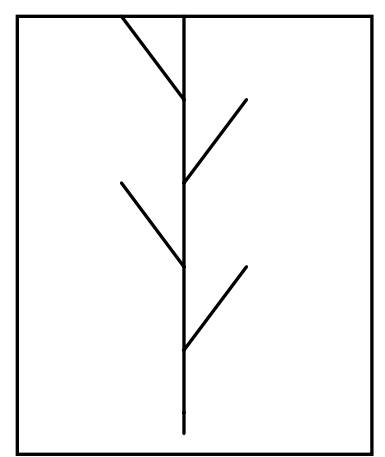

(a)

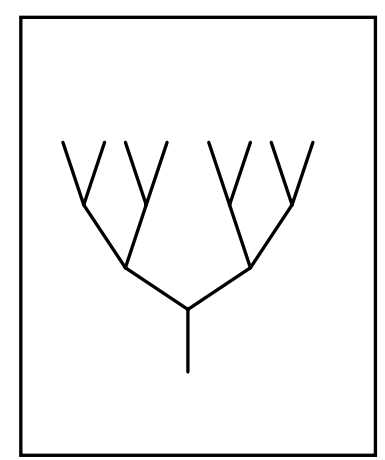

(b)

Figure 3 


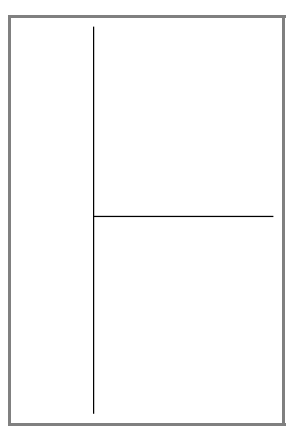

(a)

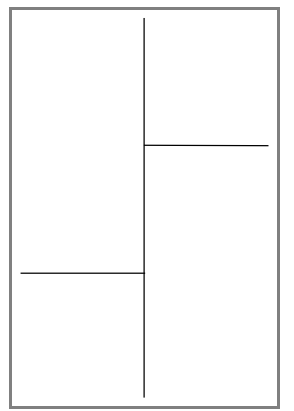

(b)

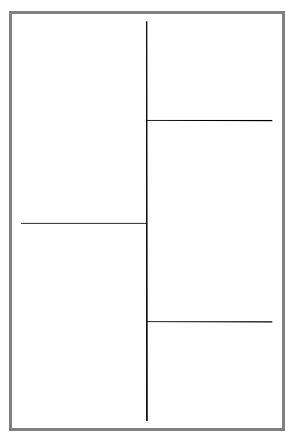

(c)

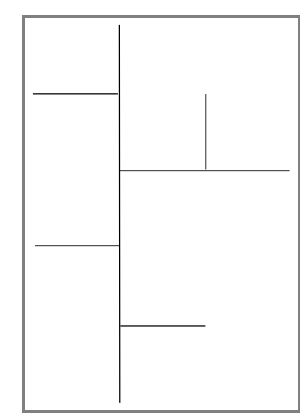

(d) 


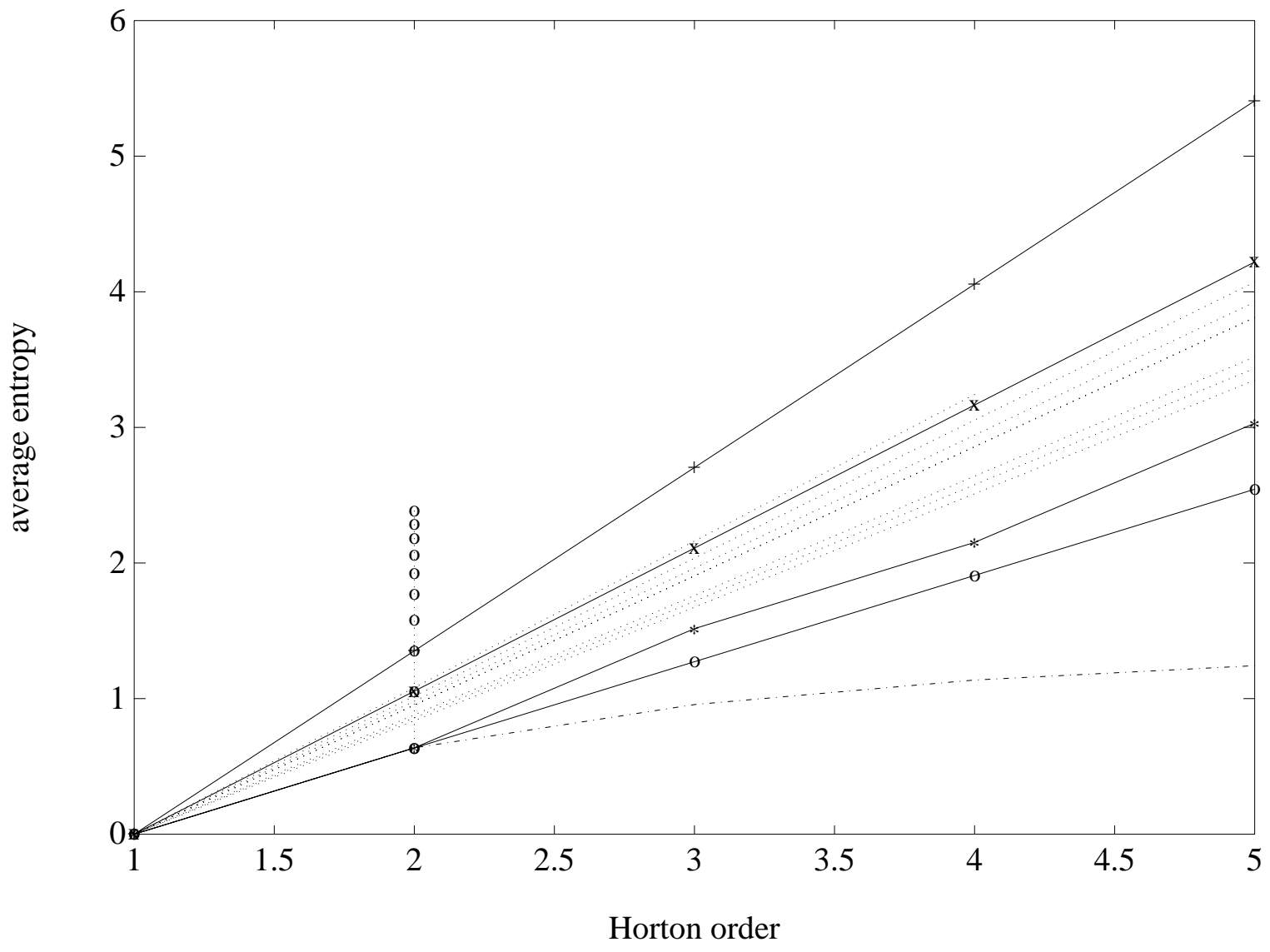

Figure 5 


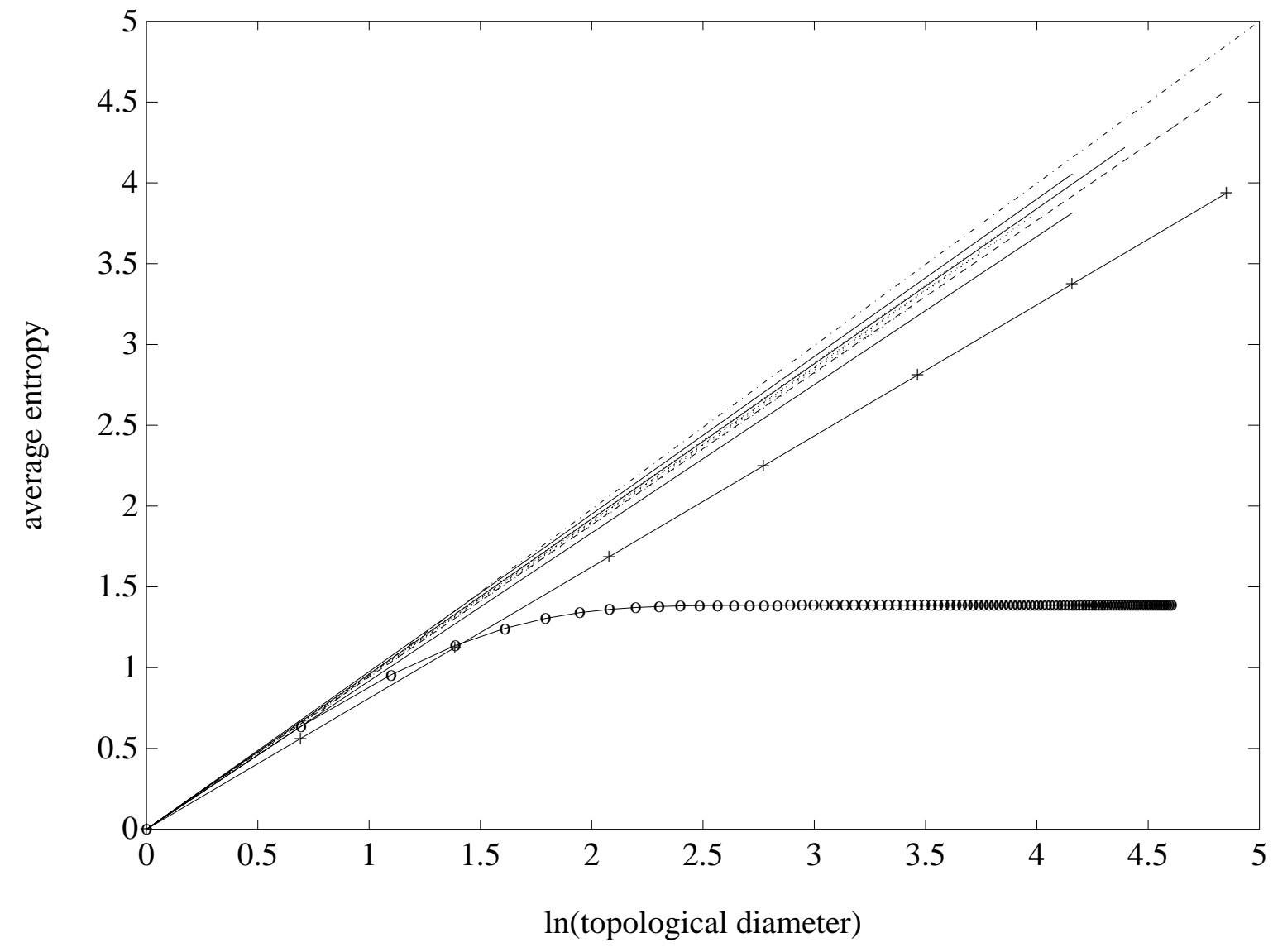

